\title{
A SEQUENCE WELL DISPERSED IN THE UNIT SQUARE
}

\author{
J. P. LAMBERT
}

(Communicated by Larry J. Goldstein)

\begin{abstract}
Distributional properties of sequences of points in the unit square have been studied extensively and there is considerable interest in sequences which are well distributed according to various criteria. Dispersion is a measure of sequence density and an important concept connected with regularity of distribution. We introduce an infinite, dyadic, easily-generated sequence which is particularly well distributed, in the sense of having dispersion of lowest possible order of magnitude.
\end{abstract}

1. Introduction. For a variety of reasons, distributional properties of sequences of points defined in the unit square have been the focus of considerable study and speculation. Particular interest has centered on measures of regularity or uniformity of distribution, and on the development of sequences well-distributed according to such measures. See, for instance $[11,12,13,15,16]$, for a small sampling of recent literature devoted specifically to regularity of distribution of points in the unit square. In a far more general sense, the paper by Niederreiter [4] is an invaluable compendium on regularity of sequence distribution and on applications of well-distributed sequences in numerical analysis.

We are concerned here with an important number-theoretic measure called dispersion. We introduce an infinite dyadic sequence in the unit square having dispersion of the best (i.e., lowest) possible order of magnitude, and having uniformly lower dispersion than any other dyadic sequence of which we are aware. In numerical practice, sequences with low dispersion are useful for quasi-random search methods, which are deterministic analogs of random search procedures for approximating global extrema of a function. Cf. $[5,6,7]$ for theoretical results on dispersion (not only in two-dimensional settings), and $[\mathbf{1}, \mathbf{8}, \mathbf{9}, \mathbf{1 0}, \mathbf{1 4}]$ for numerical experiments involving quasi-random search.

We define dispersion in the unit square $U=[0,1]^{2}$ as follows: If $\mathscr{E}_{N}$ is a set of $N$ points in $U$, then the dispersion of $\mathscr{E}_{N}$ in $U$ is

$$
d_{N}=\sup _{(\alpha, \beta) \in U} d\left((\alpha, \beta), \mathscr{E}_{N}\right)
$$

where $d$ is a totally bounded metric on $U$ and $d\left((\alpha, \beta), \mathscr{E}_{N}\right)$ means

$$
\min _{(x, y) \in \mathscr{E}_{N}} d((\alpha, \beta),(x, y))
$$

Received by the editors June 23, 1987. Presented at the International Number Theory Conference (sponsored by the Canadian Mathematical Society) at Université Laval, Québec, on July 6, 1987.

1980 Mathematics Subject Classification (1985 Revision). Primary 11K36, 11K38; Secondary $11 \mathrm{Z} 50,65 \mathrm{C} 05,65 \mathrm{C} 10$. 
Normally $d$ will denote the standard Euclidean metric in $\mathbf{R}^{2}$, although it is sometimes of interest to use the maximum metric, which we will distinguish by using $d^{\prime}$ in place of $d: d^{\prime}((\alpha, \beta),(x, y))=\max (|\alpha-x|,|\beta-y|)$. The dispersion that results from using $d^{\prime}$ will be denoted $d_{N}^{\prime}$. It should be clear that $d_{N} / \sqrt{2} \leq d_{N}^{\prime} \leq d_{N}$. If $\mathscr{E}_{N}$ is comprised of the first $N$ terms of an infinite sequence $\underline{\lambda}$ in $U$, we may denote the dispersion as $d_{N}(\underline{\lambda})$ (or as $d_{N}^{\prime}(\underline{\lambda})$ if the maximum metric is used).

Niederreiter has established lower bounds for dispersion; for $N$ points in $U$, it follows respectively from [5, Theorem 2] and from [7, Theorem 1] that

$$
d_{N} \geq \sqrt{2 / \sqrt{27} N} \quad \text { and } \quad d_{N}^{\prime} \geq 1 / 2 \sqrt{N} .
$$

So the order of magnitude of dispersion in $U$ cannot be less than $N^{-1 / 2}$.

It seems generally to be difficult to determine explicit formulas for dispersion. However, consider the Roth (or Hammersley) sequence $\left\{\left((m-1) / N, \phi_{2}(m-1)\right)\right\}_{m=1}^{N}$, where $N$ is a power of two and $\phi_{2}$ is the base two radical-inverse function; cf. [4, p. 977]. Peart [11] has determined that for this sequence, with $N=2^{k}$,

$$
d_{N}= \begin{cases}\frac{\sqrt{2 N-2 \sqrt{N}+1}}{N} & \text { for } k \text { even, } \\ \frac{\sqrt{5 N / 2-\sqrt{8 N}+1}}{N} & \text { for } k \text { odd. }\end{cases}
$$

This shows that the Roth sequence has $d_{N}=O\left(N^{-1 / 2}\right)$, the lowest possible order of dispersion. But of course the Roth sequence is finite $-N$ must be prescribed, and moreover must be a power of two.

In computational practice, it is often desirable to use infinite sequences. As Niederreiter [4, p. 972] has noted "... it is advantageous to work with an infinite sequence and then to take its first $N$ terms whenever the value of $N$ has been decided upon. In this way, we may increase $N$ if we desire greater accuracy and still use the results of the earlier computation."

2. An infinite dyadic sequence with low dispersion. We will present an infinite sequence in $U$ having low and explicitly determinable dispersion. Our sequence is also dyadic (coordinates of points are dyadic fractions) and can be rapidly generated, making it amenable to efficient computer implementation in practical numerical applications.

Sequence $\eta$ is defined as follows. Let the first term of $\eta$ be $(0,0)$. For $m=$ $1,2, \ldots$, we determine the $m+1$ st term $\left(x_{m+1}, y_{m+1}\right)$ from the $m$ th term $\left(x_{m}, y_{m}\right)$ recursively: Suppose $r$ is the smallest positive integer such that $x_{m}<1-2^{-r}$ or $y_{m} \geq 2^{-r}$. Then, if $x_{m}<1-2^{-r}$, let $x_{m+1}=x_{m}+3 \times 2^{-r}-1$ and $y_{m+1}=$ $y_{m}+\gamma \times 2^{-r}$, where $\gamma=1$ if $y_{m}<2^{-r}$ and $\gamma=-1$ if $y_{m} \geq 2^{-r}$. Otherwise let $x_{m+1}=x_{m}+2^{-r}-1$ and $y_{m+1}=y_{m}$

The sequence $\eta$ beings with the points

$$
\begin{aligned}
(0,0),\left(\frac{1}{2}, \frac{1}{2}\right),\left(0, \frac{1}{2}\right),\left(\frac{1}{2}, 0\right) & ,\left(\frac{1}{4}, \frac{1}{4}\right), \\
& \left(\frac{3}{4}, \frac{3}{4}\right),\left(\frac{1}{4}, \frac{3}{4}\right),\left(\frac{3}{4}, \frac{1}{4}\right),\left(0, \frac{1}{4}\right),\left(\frac{1}{2}, \frac{3}{4}\right), \ldots
\end{aligned}
$$




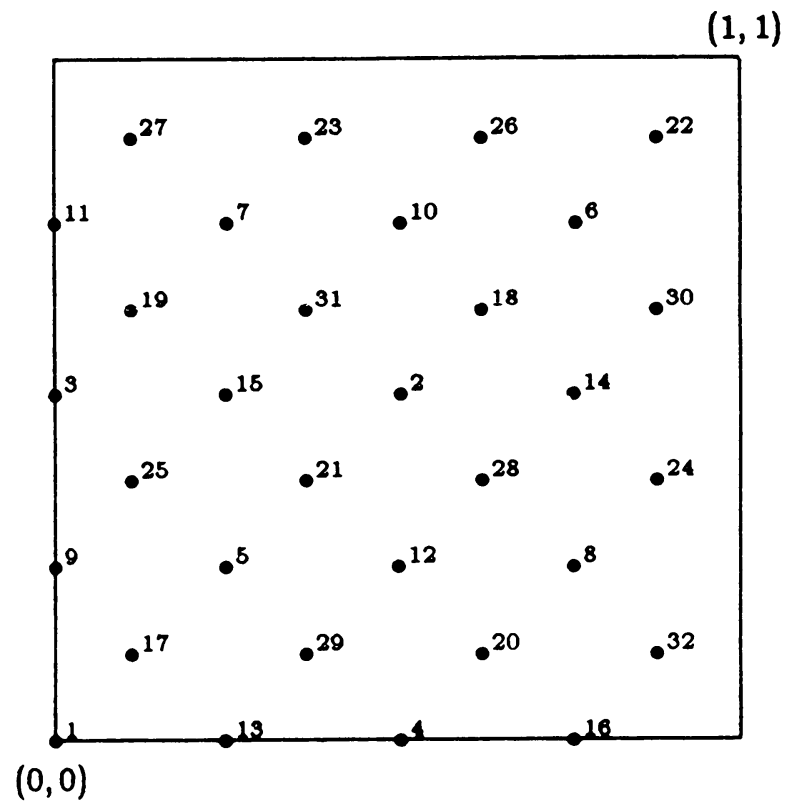

Figure 1. The first 32 terms of $\eta$ in $U=[0,1]^{2}$

The first 32 terms are depicted in Figure 1. If $k$ is odd, the first $2^{k}$ terms of $\eta$ fill out a "diagonal" lattice (as in Figure 1); if $k$ is even, the first $2^{k}$ terms of $\eta$ fill out a regular $2^{k / 2}$ by $2^{k / 2}$ square lattice (consider, e.g. the first 16 terms in Figure 1). Notice that the 17 th through the 32 nd terms are the centers of the 16 small squares delineated by the first 16 terms (including here the "boundary squares" for which an edge lies along the line $x=1$ or the line $y=1$ ).

A more intuitive geometric construction of the sequence $\eta$ is possible [3] using a two-dimensional "splitting and stacking" technique, a two-dimensional version of a scheme due to von Neumann and Kakutani for constructing ergodic measurepreserving transformations on the unit interval. The use of one-dimensional splitting and stacking to generate well-distributed sequences in the unit interval is discussed in [2].

Although it turns out that $\eta$ is itself nicely dispersed in $U$, we can improve matters, obtaining slightly lower dispersion, if we simply change the first term from $(0,0)$ to $(1,1)$; let this new sequence be denoted $\hat{\eta}$. The first $N$ terms of $\eta$ and of $\hat{\eta}$ will be denoted by $\mathscr{E}_{N}$ and $\hat{\mathscr{E}}_{N}$ respectively. We next proceed to determine the dispersion of $\hat{\eta}$.

First suppose $k$ is an even positive integer. In this case $\mathscr{E}_{2^{k}}$ is the square lattice $\left\{\left(i \times 2^{-k / 2}, j \times 2^{-k / 2}\right): i, j=0,1, \ldots, 2^{k / 2}-1\right\}$, and $\hat{\mathscr{E}}_{2^{k}}=\mathscr{E}_{2^{k}} \cup\{(1,1)\}-\{(0,0)\}$. Now the point $\mathbf{a}=\left(1,2^{-k / 2-1}\right) \in U$ is at least as distant from its closest neighbor in $\hat{\mathscr{E}}_{2^{k}}$ (which we can take to be the point $\mathbf{b}=\left(1-2^{-k / 2}, 0\right)$ ) as is any other point of $U$ from its closest neighbor in $\hat{\mathscr{E}}_{2^{k}}$ (see Figure 2a). So $d_{2^{k}}=d(\mathbf{a}, \mathbf{b})=\sqrt{5 / 2^{k+2}}$. For $N=2^{k}+1,2^{k}+2, \ldots, 2^{k+1}-1, \mathscr{E}_{N}$ is formed from the lattice $\hat{\mathscr{E}}_{2^{k}}$ by including points at the centers of the squares of the lattice, as well as centers of the "boundary squares" lying along the boundaries $x=1$ and $y=1$, with the exception of the square at the lower right corner of $U$ (the square having points $\mathbf{b}$ and $(1,0)$ as 


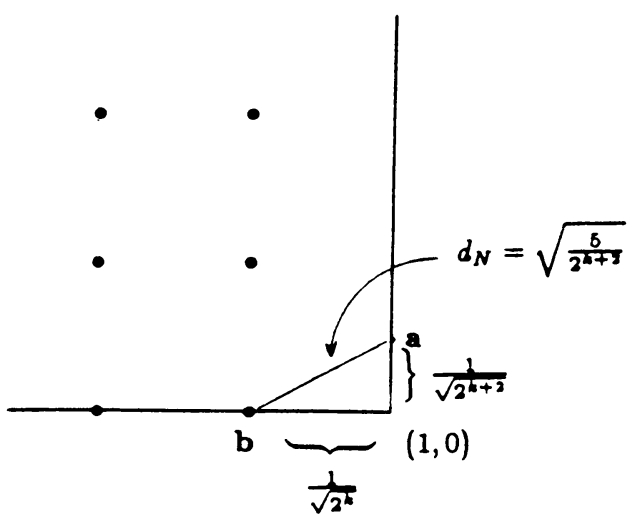

FIGURE 2a. $N=2^{k}, k$ even

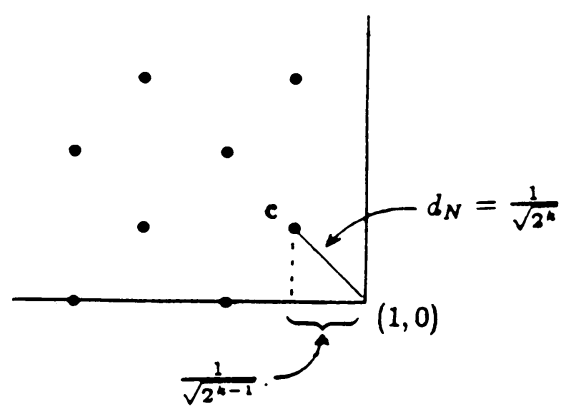

FIGURE 2b. $n=2^{k}, k$ odd

adjacent vertices, the center of which is the $2^{k+1} s t$ term of $\hat{\eta}$ ). Formally,

$$
\begin{aligned}
\hat{\mathscr{E}}_{2^{k+1}-1}= & \mathscr{E}_{2^{k}} \cup\left\{\left(i \times 2^{-k / 2-1}, j \times 2^{-k / 2-1}\right): i, j=1,3, \ldots, 2^{k / 2+1}-1\right\} \\
& -\left\{\left(1-2^{-k / 2-1}, 2^{-k / 2-1}\right)\right\} .
\end{aligned}
$$

And so for these values of $N$ we also have $d_{N}=d(\mathbf{a}, \mathbf{b})=\sqrt{5 / 2^{k+2}}$.

Next suppose $k$ is an odd integer. In this case $\mathscr{C}_{2^{k}}$ is a $2^{(k-1) / 2}$ by $2^{(k-1) / 2}$ square lattice augmented with midpoints of lattice squares (including "boundary squares" along $x=1$ and $y=1$ ). Formally,

$$
\begin{aligned}
& \mathscr{E}_{2^{k}}=\left\{\left(i \times 2^{-(k+1) / 2}, j \times 2^{-(k+1) / 2}\right): i, j=0,1, \ldots, 2^{(k+1) / 2}-1 ; i+j \text { even }\right\} \text { and } \\
& \hat{\mathscr{E}}_{2^{k}}=\mathscr{E}_{2^{k}} \cup\{(1,1)\}-\{(0,0)\} .
\end{aligned}
$$

Here the point $(1,0) \in U$ is at least as distant from its closest neighbor in $\hat{\mathscr{E}}_{2^{k}}$ (which we can take to be the point $\mathrm{c}=\left(1-2^{-(k+1) / 2}, 2^{-(k+1) / 2}\right)$ ) as is any other point of $U$ from its closest neighbor in $\hat{\mathscr{E}}_{2^{k}}$ (see Figure 2b). So $d_{2^{k}}=d((1,0), \mathrm{c})=2^{-k / 2}$. For $N=2^{k}+1,2^{k}+2, \ldots, 2^{k+1}-1, \hat{\mathscr{E}}_{N}$ continues to fill in a $2^{(k+1) / 2}$ by $2^{(k+1) / 2}$ square lattice by picking up all points $\left(i \times 2^{-(k+1) / 2}, j \times 2^{-(k+1) / 2}\right)$ with $i, j=$ $0,1, \ldots, 2^{(k+1) / 2}-1$ for which $i+j$ is odd, excepting the point $\left(1-2^{-(k+1) / 2}, 0\right)$, which is the $2^{k+1}$ st term of $\hat{\eta}$. None of these additional points is as close to $(1,0)$ as is $\mathrm{c}$, and so for these values of $N$ we continue to have $d_{N}=2^{-k / 2}$. We have established

THEOREM 1. Let the sequence $\hat{\eta}$ have first term $(1,1)$ and be otherwise identical with $\eta$. Then, for $k=1,2, \ldots$ and $2^{k} \leq N<2^{k+1}$,

$$
d_{N}(\hat{\eta})= \begin{cases}\sqrt{5 / 2^{k+2}} & \text { for } k \text { even } \\ 1 / \sqrt{2^{k}} & \text { for } k \text { odd }\end{cases}
$$


In particular, if $N=2^{k}$, then

$$
d_{N}(\hat{\eta})= \begin{cases}\sqrt{5 / 4 N} & \text { for } k \text { even } \\ 1 / \sqrt{N} & \text { for } k \text { odd }\end{cases}
$$

implying that $\hat{\eta}$ has smaller dispersion than has the Roth sequence for all $N(>1)$ for which the latter is defined. A routine consequence of Theorem 1 is

COROllary. For $N=2,3, \ldots, 1 / \sqrt{N} \leq d_{N}(\hat{\eta}) \leq \sqrt{5 /(2 N+2)}$.

The inequalities in the corollary are best possible in the sense that there is equality on the left if $N=2^{k}$ with $k$ odd, and there is equality on the right if $N=2^{k}-1$ with $k$ odd.

Although we will not go through the details here, a rather similar analysis can be used for the sequence $\eta$; it turns out that, for $k$ odd,

$$
d_{N}(\eta)= \begin{cases}1 / \sqrt{2^{k}} & \text { if } 2^{k} \leq N \leq\left(2^{k+3}-1\right) / 3 \\ \sqrt{5 / 2^{k+3}} & \text { if }\left(2^{k+3}-1\right) / 3<N<2^{k+2} .\end{cases}
$$

This implies that $1 / \sqrt{N} \leq d_{N}(\eta) \leq \sqrt{8 /(3 N+1)}$, with equality on the left if $N=2^{k}$ ( $k$ odd), and on the right if $N=\left(2^{k+3}-1\right) / 3$ ( $k$ odd).

We now consider the dispersion $d_{N}^{\prime}$ for the sequence $\eta$ (the situation for $\hat{\eta}$ is identical).

THEOREM 2. If $k$ is odd and $2^{k} \leq N<2^{k+2}$, then $d_{N}^{\prime}(\eta)=1 / \sqrt{2^{k+1}}$.

ProOF. Suppose $k$ is odd. Then, for $N=2^{k}$, as we have noted in proving Theorem 1,

$$
\mathscr{C}_{N}=\left\{\left(i \times 2^{-(k+1) / 2}, j \times 2^{-(k+1) / 2}\right): i, j=0,1, \ldots, 2^{(k+1) / 2}-1 ; i+j \text { even }\right\} .
$$

Consider the point $(1,0) \in U$. For $(x, y) \in \mathscr{E}_{N}, d^{\prime}((1,0),(x, y))=\max (1-x, y)$; and this value is minimized by taking $(x, y)=\left(1-2^{-(k+1) / 2}, 2^{-(k+1) / 2}\right)=\mathrm{c}$ (see Figure $2 \mathrm{~b})$, the minimum value being $2^{-(k+1) / 2}$. So $d^{\prime}\left((1,0), \mathscr{E}_{N}\right)=d^{\prime}((1,0), \mathrm{c})=$ $2^{-(k+1) / 2}$. If $(\alpha, \beta)$ is any other point in $U$, then it should be clear (again, see Figure $2 \mathrm{~b})$ that $d^{\prime}\left((\alpha, \beta), \mathscr{E}_{N}\right) \leq 2^{-(k+1) / 2}$. Hence $d_{N}^{\prime}=2^{-(k+1) / 2}$. Now for $N=2^{k}+1,2^{k}+2, \ldots, 2^{k+2}-1$, the square at the lower right corner of $U$ having diagonally opposite vertices $c$ and $(1,0)$ is filled in (with terms of $\eta$ ) only to the extent that the lower left vertex $\left(1-2^{-(k+1) / 2}, 0\right)$ is added, and so we still have $d^{\prime}\left((1,0), \mathscr{E}_{N}\right)=d^{\prime}((1,0), \mathrm{c})=2^{-(k+1) / 2}$.

COROLLARY. $1 / \sqrt{2 N} \leq d_{N}^{\prime}(\eta) \leq \sqrt{2 /(N+1)}$.

There is equality on the left side in the corollary if $k$ is odd and $N=2^{k}$, equality on the right side if $k$ is odd and $N=2^{k}-1$.

In closing, it should perhaps be noted that dispersion is related to a very widely used measure of uniformity known as discrepancy. If $D_{N}$ is the discrepancy of a set of $N$ points $\mathscr{E}_{N}$ in $U$ then, as Niederreiter [5, Theorem 3; and 6, p. 1166] has shown, $d_{N} \leq \sqrt{2 D_{N}}$ and $d_{N}^{\prime} \leq \sqrt{D_{N}}$. For the definition of $D_{N}$ see [4, Definition 2.6]. Discrepancy is important in numerical analysis because of its role in quasiMonte Carlo integration theory (cf. [4]). We conjecture that if $\mathscr{E}_{N}$ is comprised of the first $N$ terms of $\eta$ then $(2 \sqrt{N}-1) / 4 N<D_{N}<(8 \sqrt{N}-4) / N$. 


\section{REFERENCES}

1. T. J. Aird and J. R. Rice, Systematic search in high dimensional sets, SIAM J. Numer. Anal. 14 (1977), 296-312.

2. J. P. Lambert, Quasi-Monte Carlo, low discrepancy sequences, and ergodic transformations, J. Comput. Appl. Math. 12/13 (1985), 419-423.

3. __ On the development of infinite low-discrepancy sequences for quasi-Monte Carlo implementation, Technical Report, University of Alaska, Fairbanks, March 1986.

4. H. Niederreiter, Quasi-Monte Carlo methods and pseudo-random numbers, Bull. Amer. Math. Soc. 84 (1978), 957-1041.

5. __ A quasi-Monte Carlo method for the approximate computation of the extreme values of a function, Studies in Pure Mathematics (To the Memory of Paul Turán), Birkhäuser, Basel, 1983, pp. 523-529.

6. __ On a measure of denseness for sequences, Topics in Classical Number Theory, Colloq. Math. Soc. János Bolyai, 34, (Budapest, 1981), North-Holland, Amsterdam, 1984, pp. 11631208.

7. __ Quasi-Monte Carlo methods for global optimization, Proc. 4th Pannonian Sympos. on Math. Stat., (Bad Tatzmannsdorf, Austria, 1983), Akad. Kiado, Budapest, 1986, pp. 251267.

8. H. Niederreiter and K. McCurley, Optimization of functions by quasi-random search methods, Computing 22 (1979), 119-123.

9. H. Niederreiter and P. Peart, A comparative study of quasi-Monte Carlo methods for optimization of functions of several variables, Caribbean J. Math. 1 (1982), 27-44.

10. __ Localization of search in quasi-Monte Carlo methods for global optimization, SIAM J. Sci. Stat. Comput. 7 (1986), 660-664.

11. P. Peart, The dispersion of the Hammersley sequence in the unit square, Monatsh. Math. 94 (1982), 249-261.

12. R. G. E. Pinch, A sequence well distributed in the square, Math. Proc. Cambridge Philos. Soc. 99 (1986), 19-22.

13. K. F. Roth, On irregularities of distribution. II, Comm. Pure Appl. Math. 29 (1976), 749-754.

14. I. M. Sobol and R. B. Stanikov, LP-search and problems of optimal design, Problems of Random Search, vol. 1, Izdat. "Zinatne," Riga, 1972, pp. 117-135 (Russian).

15. S. Srinivasan, On two-dimensional Hammersley's sequences, J. Number Theory 10 (1978), 421-429.

16. B. E. White, On optimal extreme-discrepancy point sets in the square, Numer. Math. 27 (1977), 157-164.

Department of Mathematical Sciences, University of Alaska, Fairbanks, ALASKA 99775-1110 